hip, and soreness about the body. The bowels have acted; the pulse is 80 ; tongue slightly furred.

20. This morning Mr. Parker, the house-surgeon, found the patient with symptoms of coma; it was with difficulty he could be roused, breathing heavily, with slow pulse. In consequence of these appearances we had the head shaved, and made a careful examination; a small tumor of effused blood was found over the left temple; slight ecchymosis was observed over the upper and lower eyelids of the left side; there was also a small spot on the conjunctiva of the left eye; there is paralysis of the left side of the face. On pressing the tumor over the temple, he shows from his movements that it gives pain; on speaking loudly and shaking him, he answers in monosyllables; the pupils are dilated; they act slightly. Cold lotion was ordered to the head; turpentine enemata were ordered, and three grains of calomel, with one and a half of opium every two hours.

21. The symptoms continue without any alteration; the bowels have been freely opened; the symptoms of coma are not increased.

22. The gums are affected by the mercury; the symptoms of compression are not increasing. Omit the calomel.

It is unnecessary to give the daily symptoms in this case s suffice it to say, that there was a rapid improvement manifested each day, and at the expiration of fourteen days from the attack the symptoms en. tirely disappeared, the patient continuing well under the influence of mercury the whole time.

\section{HYDATIDS OF THE UTERUS.}

TO THE EDITORS OF THE PROVINCIAL MEDICAL JOURNAL.

Gentlemen,-I consider that every medical man ought, as far as he can, make known to his medical brethren any case of practical value that may come under his observation, and more especially that every member of the Provincial Medical and Surgical Association should publish such in your excellent Journal. This being my opinion, I send you the following case of hydatids of the uterus, which, if you consider of sufficient interest, shall be obliged by your insertion of it in the Journal :-

On the 8 th of December last I was sent for, about three, a.m., to attend Mrs. H., of this place, then in labor. On my arrival I found the presentation natural, and delivery took place in about an hour; in fact, the labor was a common-place one, and the patient went on well till the 20 th, when her husband came in a great hurry, desiring me to see his wife immediately, as he was afraid she was dying. I hastened to her house, and found her in a state of syncope. Profuse hæmorrhage had taken place, and was still continuing; two chamber utensils had been emptied prior to my arrival; the one I saw was more than half full, and the bed-clothes were saturated with blood. I immediately ordered cloths wet with vinegar and water to be applied over the abdomen, loins, thighs, \&c., while I went for some ammonia and opium, which might have taken me about ten minutes. On my return I found that the hæmorrhage had entirely ceased, and on giving her the stimulant she rallied.
I now made inquiries into the particulars of the case. It appeared that she got out of bed in the morning, and on returning found something running away from her, and directly she felt a substance come away, which, on examination I found to be a mass of hydatids. I cannot describe the size better than in the words of a woman present--"Why, Sir, it is as large as a child's head." I called again in the course of the day, and found there had been no return of the hæmorrhage, and in a few days the patient was down stairs quite recovered, except being weak from the great loss of blood.

I beg to remark two circumstances in this case which I consider rather extraordinary; first, the manner in which the hydatids came off (I cannot say expelled), for it appears there was no uterine contraction; it must have been from their own weight; secondly, the sudden stoppage of the hæmorrhage, for, after the cold application, there was not sufficient to stain a napkin.

I am, Gentlemen, Your obedient servant, F. A. Clewe.

Bradninch, Jan. 14, 1843.

\section{REMARKS ON CHOLERA.}

TO THE EDITORS OF THE PROVINCIAL MEDICAL JOURNAL.

Gentlemen,-The subject which now claims attention is the state of the circulation in cholera, and it may be very advantageously divided into two heads - viz., the action of the organs by which the circulation is carried on, and the condition of the circulating fluid.

1st. Of the action of the heart and arteries. From what has been already said, it will be inferred that the heart and arteries are impaired in action, whether the force or frequency of their contractions be regarded, and in this respect correspond most remarkably with the respiration. From what was stated in a former letter, it might be supposed that this state of the circulation was to be imputed solely to the imperfectly oxygenated blood supplied to the heart, owing to the defective action of the lungs; but in cholera other circumstances exist, the tendency of which is to prohibit such an exclusive explanation. No fact in physiology or pathology is better known, or more easy of demonstration, than the mutual dependence on each other of the respiratory and circulatory systems-in other words, the normal action of the lungs is essential to healthy circulation, and so is a healthy condition of the latter to the function of respiration. Hence it is, that where the heart is diseased the respiratory system is sooner or later a participator; and where the function of respiration is materially disordered, such derangement almost immediately implicates the circulation. In such cases, however, one system is unequivocally affected in consequence of the deranged state of the other; and whilst the action of the one is increased that of the other may be absolutely diminished, and vice versi -in fact they may be regarded simply as cause and effect. What, then, it may be asked, is the peculiarity connected with these systems in cholera? Is it that, so far as my observation extends, the most perfect correspondence between the 